\title{
Des sujets aux citoyens, une analyse des projets electoraux avant les cortes de cadix, 1808-1810
}

\section{Richard Hocquellet}

\section{(2) OpenEdition \\ 1 Journals}

Édition électronique

URL : https://journals.openedition.org/ahrf/306

DOI : 10.4000/ahrf.306

ISSN : 1952-403X

Éditeur :

Armand Colin, Société des études robespierristes

Édition imprimée

Date de publication : 1 mars 2001

Pagination : 1-19

ISSN : 0003-4436

Référence électronique

Richard Hocquellet, «Des sujets aux citoyens, une analyse des projets electoraux avant les cortes de cadix, 1808-1810", Annales historiques de la Révolution française [En ligne], 323 | janvier-mars 2001, mis en ligne le 16 mars 2006, consulté le 23 avril 2022. URL : http://journals.openedition.org/ahrf/306 ; DOI : https://doi.org/10.4000/ahrf.306

Ce document a été généré automatiquement le 23 avril 2022.

Tous droits réservés 


\title{
Des sujets aux citoyens, une analyse des projets electoraux avant les cortes de cadix, 1808-1810
}

\author{
Richard Hocquellet
}

1 Suite aux abdications de la famille royale en faveur de Napoléon en mai1808, les Espagnols qui luttent contre les Français doivent s'organiser politiquement pour exister en tant que pouvoir réel face au gouvernement de Joseph Bonaparte. La solution à la disparition de ce que les patriotes considéraient comme le pouvoir légitime, ne s'impose pas de suite. On passe par des tentatives dont les juntes suprêmes provinciales, puis la Junte centrale et le Conseil de régence sont les étapes. Elles provoquent de multiples réflexions sur le type de pouvoir qu'exercent de fait les patriotes et sur leur mode de représentation. Elles aboutissent finalement à la réunion d'une Assemblée constitutante, les Cortes de Cadix, en septembre 1810.

2 Si la nécessité de les réunir n'a pratiquement pas été remise en cause, la manière de les convoquer a donné lieu à un des débats les plus riches de la période. La toile de fond événementielle est occupée par la guerre contre les armées napoléoniennes qui soutiennent le projet impérial de faire de l'Espagne un État vassal. Dès juin 1808, Napoléon a doté la Monarchie catholique d'une constitution qui établit la séparation des pouvoirs, le pouvoir législatif revenant à des Cortes refondées. Les patriotes qui refusent la solution imposée par la France à la crise du régime ne se battent pas pour autant pour la permanence d'un système qui vient de prouver sa faillite. Le déroulement $\mathrm{du}$ soulèvement patriotique dans les provinces libres de troupes impériales entre la fin du mois de mai et le début de celui de juin 1808 provoque une rupture majeure dans les relations qu'entretiennent les Espagnols avec le pouvoir. En décidant la révolte vis-à-vis du gouvernement légal de Madrid contrôlé par Murat au nom de la défense du souverain légitime Ferdinand VII, les patriotes font valoir un fondement différent du pouvoir qui se traduit par l'institution des juntes suprêmes (au sens de souveraines). Ces organes de gouvernement locaux, qui s'entendent au mois de septembre 1808 pour mettre en place la Junte centrale, revendiquent leur autorité au 
nom de principes qui bouleversent les bases de la monarchie bourbonienne le « vœu de la nation plutôt que le fait du prince ${ }^{1}$. À partir de là, un processus s'engage de mutations profondes dans l'ordre du politique durant les premières années de la Guerre d'Indépendance espagnole.

C'est à travers l'étude des projets électoraux parus en préparation de cette convocation que je me propose de les analyser.

4 L'intérêt de ces textes par rapport à l'ensemble du discours patriotique réside dans le fait qu'ils révèlent un effort de cohérence dans la pensée du politique. En mettant au point des systèmes électoraux, les auteurs s'obligent à transposer dans la pratique des théories. Inversement, l'analyse de ces projets renseigne sur la réalité et la validité des idées énoncées ainsi que leurs implications concrètes. Prendre les élections comme objet historique nous amène à être plus attentifs aux modalités de l'action politique ${ }^{2}$.

Pour la période comprise entre juillet 1808 et septembre 1810, j'ai pu rassembler 66 textes contenant des projets électoraux. La plupart proviennent de la consultation nationale (consulta al pais) lancée par la Junte centrale suite au décret du 22 mai 1809 annonçant la prochaine convocation des Cortes. Ils ont la forme de rapports envoyés à

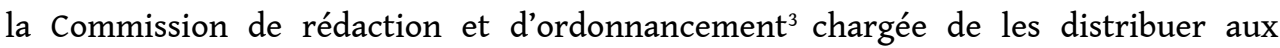
différentes commissions préparatoires aux Cortes (28 dictamenes oficiales et 11mémoires individuels $\left({ }^{4}\right)$ ). D'autres sont des mémoires de particuliers, au nombre de 6 , commandés par la junte de Catalogne et devant servir de base à l'élaboration de son rapport ${ }^{5}$. Par ailleurs, le recensement des textes patriotiques politiques fait apparaître des écrits traitant de ces mêmes thèmes. Ils sont soit anonymes [10], soit signés [11], publiés en feuillets ou dans la presse et correspondent à des prises de positions spontanées dans le débat du moment ${ }^{6}$.

La distribution chronologique de ces textes entre l'été 1808 et l'automne 1810 n'est pas égale. Elle s'aligne sur la chronologie des étapes de la vie politique de l'Espagne comme nous pouvons le voir sur le graphique suivant:

Distribution chronologique

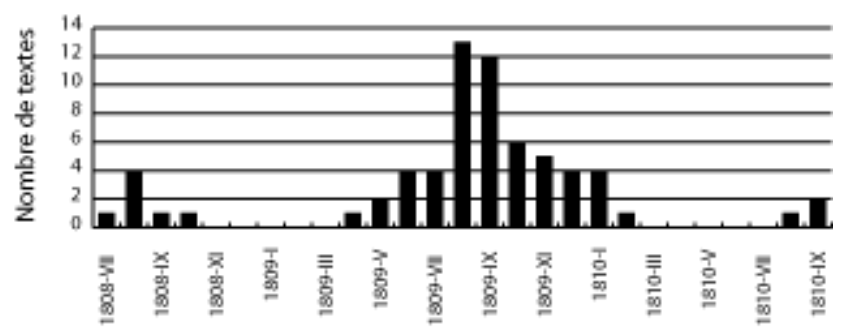

7 Trois moments bien isolés sont repérables. Ils nous aident à saisir la motivation des auteurs et à définir leur problématique.

Le premier moment se situe en juillet, août et septembre 1808, cet été du soulèvement patriotique où, après la formation des juntes suprêmes qui reprennent en main l'autorité dans les provinces et se chargent d'organiser la lutte, les patriotes cherchent à établir un pouvoir central représentant tout autant celui du roi absent que l'idéal patriotique. Certains des textes écrits à ce sujet vont aller plus loin que l'organisation d'un gouvernement de circonstance et proposer une refonte du système politique, seul moyen d'assurer légitimité et stabilité à ce nouveau pouvoir.

9 Les mois qui suivent voient disparaître ce genre d'écrits. La Junte centrale a été reconnue par les provinces patriotes. La relance du débat commence au début de l'été 
1809 à l'initiative de la Junte centrale, suite à son décret du 22 mai 1809 qui assure les Espagnols de la prochaine réunion des Cortes. C'est le moment de la consultation nationale dont les premières réponses arrivent en juillet. Elles vont s'étaler jusqu'en décembre et constituer un ensemble de réflexions sur la monarchie espagnole sans précédent.

Le troisième moment correspond aux quelques mois qui précèdent l'ouverture des Cortes. Suite à l'invasion de Séville par les troupes napoléoniennes en janvier 1810, le gouvernement central s'est replié à Cadix. Dans les derniers réduits de l'Espagne patriote des auteurs vont faire publier des textes qui se présentent comme des bases de discussion pour les futures Cortes, comme des projets dont pourraient s'inspirer les députés.

11 Au-delà de la diversité des manières d'exposer les idées, l'ensemble de ces textes peut s'étudier comme un système articulé autour de quelques questions essentielles. J'en retiendrai plus particulièrement deux: d'une part celle de la définition du corps politique et d'autre part celle de sa participation à l'action politique dont la première modalité correspond au droit d'élire.

La vision du corps politique, nouvelles approches de la nation

Définir ou redéfinir le corps politique revient à poser la question de la nation, sa composition et les liens qui permettent d'inclure ses membres dans l'ordre politique.

Tout d'abord voyons comment le discours sur la nation énonce une nouvelle vision $\mathrm{du}$ corps politique

La maison commune, la métaphore familiale du corps politique

grand élan du soulèvement de mai et juin 1808 s'est produit grâce au mouvement populaire. Le rôle joué par le peuple (bajo pueblo, plebe) dans la résistance à l'occupation française a été une des composantes du patriotisme espagnol. Le discours s'en est fait l'écho dès ses premières productions. Parallèlement le soulèvement fut reconnu unanime dans toutes les provinces. Les patriotes en tirent deux conclusions immédiates. Premièrement le peuple ayant montré l'exemple il doit être considéré non plus comme la partie la plus basse de la population (bajo pueblo) mais comme la partie la plus nombreuse de la population (el Pueblo español). Les expressions employées à l'époque sont étonnantes en regard de ce qu'était la monarchie espagnole quelques semaines plus tôt :

«Ce bas peuple qui est la partie constitutive des États et le dépositaire le plus sûr de nos anciennes vertus publiques.» ${ }^{7}$

La deuxième conclusion prend en compte cet aspect et le met en parallèle avec la reconnaissance de l'identité patriotique dans toutes les provinces. Chaque province s'est d'abord soulevée isolément, autour de sa capitale. Les nouvelles du soulèvement de toutes les provinces non occupées par les Français font prendre conscience qu'il existe une communauté de sentiments et une équivalence du mouvement.

«Il n'y a plus maintenant en Espagne des Estrémègnes, des Andalous, des Valenciens, des Aragonais ni d'autres provinces, il n'y a seulement que des Espagnols et à ce grand corps politique une seule âme donne vie.» ${ }^{8}$

16 Nous pouvons observer un glissement dans la représentation politique du peuple : les patriotes s'identifient à l'ensemble du peuple pour aboutir à une nouvelle équation le peuple=la nation au sens d'une communauté constituée autour d'un même intérêt politique ${ }^{9}$. 
17 Le poète Manuel Quintana, futur animateur des libéraux à la Junte centrale, explicite ce glissement dans les premiers numéros du Semanario patriótico. Il en arrive à la conclusion que le mouvement qui s'est opéré en Espagne depuis le soulèvement revient à « fonder une patrie » car les Espagnols sont devenus des « frères d'armes ». Il s'agit là d'une patrie-fratrie selon une métaphore familiale qui renvoie à une vision traditionnelle de la communauté. La métaphore se retrouve aussi dans la manière de considérer la place du roi : si les Espagnols sont tous frères, ils ont le même père : le roi Ferdinand VII ; ce qui leur fait dire, suite à son abdication forcée qu'ils sont des «orphelins ». Dans la logique de cette vision de la nation espagnole, les patriotes précisent que personne, aucune catégorie, ne peut prétendre incarner à elle seule la nation.

18 Les deux fondements de la nation moderne se retrouvent dans le mouvement patriotique le droit des peuples et le principe des nationalités ${ }^{10}$. En refusant les abdications, les Espagnols revendiquent le droit de prendre en main leur destin et en luttant contre l'occupant français, ils s'appuient sur une identité nationale déjà définie dans le champ culturel et historique ${ }^{11}$.

La nation à l'épreuve du politique

Pour l'instant, j'ai pu me permettre de ne pas prendre en considération les différentes acceptions des termes peuple, nation, patrie car les auteurs eux-mêmes finissent par les interchanger. Mais, quand il va s'agir d'aborder les conséquences politiques de cette nouvelle approche de la nation espagnole, ces termes vont revêtir des sens différents selon l'opinion des auteurs. Des divergences apparaissent à propos de la définition des capacités des membres de la nation et des procédures qui permettent de se reconnaître comme participant à un même corps politique.

"Au peuple seul se doit la liberté de la nation $»^{12}$. Cette affirmation largement partagée sert de base aux nouveaux principes qui impliquent une inclusion de ce peuple dans le corps politique. Reste à savoir s'il devient la seule et unique référence pour le constituer ou s'il gagne seulement en considération à côté de la noblesse et du clergé qui y possédaient jusqu'alors plus de droits. On s'aperçoit qu'il n'est pas toujours considéré comme le rassemblement de tous les membres de cette nation. Beaucoup de textes le considèrent encore comme le troisième ordre, le commun (el común).

21 Le débat porte sur la question des privilèges. Dans l'ancienne vision de la nation, la qualité de membres du corps politique était fonction de privilèges : ceux de la noblesse divisée en trois catégories (grandes de España, titulados de Castilla, hidalgos), ceux du clergé, divisé entre prélats et autres clercs, et ceux de quelques villes ayant droit à une représentation aux Cortes. Ces privilèges sont tous considérés comme abusifs, traces d'un temps aujourd'hui révolu.

Cette critique des privilèges ne débouche pas sur une même définition du nouveau corps politique. On observe une déclinaison de propositions allant du respect de la séparation en ordres jusqu'à l'affirmation d'une communauté d'individus égaux.

Les auteurs les plus traditionnels respectent les divisions anciennes: la nation est constituée des trois ordres, chaque membre de la nation se rattache exclusivement à l'un d'eux. L'intégration du peuple - au sens du tiers état- au corps politique se fait par l'intermédiaire des villes, c'est-à-dire des institutions municipales qui puisent leur légitimité politique dans l'héritage médiéval. Ce type de propositions apparaît surtout sous la plume de membres du clergé attachés à la tripartition justifiée par l'Église 
depuis le Moyen Âge. Les 19 textes recensés (près de 30\%) gardant une vision par ordres du corps politique font preuve de prudence vis-à-vis des événements, ils craignent tous une modification trop brutale de l'organisation politique. Ils se réfèrent aussi aux vieux codes de lois, voire aux constitutions médiévales.

D'autres textes, moins nombreux [13], tentent d'aménager cette tradition en tenant compte des circonstances. Le corps politique est toujours pensé à partir des distinctions d'ordres mais la part que doit y tenir le peuple est augmentée pour rendre justice à ses efforts dans la lutte. L'idée est que «tout le peuple soit entendu $»^{13}$ (selon l'expression de l'audience d'Estrémadure dans son rapport sur les Cortes).

Un pas est franchi avec la disparition de la distinction d'ordres ou de classes. On pense alors en terme d'individus : tous les Espagnols sont mis sur un pied d'égalité.

Pour autant, certains auteurs veulent préserver une certaine hiérarchie dans ce corps politique si vaste et si égal. Des constructions souvent artificielles sont présentées pour distinguer ses membres. Certains auront plus de droits que d'autres. Ainsi de la représentation aux Cortes: certes tous les membres du corps politique pourront participer aux élections mais on choisira d'avantager certains. Soit les archevêques, membres de droit des Cortes. Soit les élites intellectuelles et économiques par de savants systèmes de péréquation visant à augmenter la représentation des grandes villes : les "talents doivent représenter la masse du peuple »" Soit en faisant une différence selon la fortune : seuls les plus riches pourront être élus. Le recours à un système censitaire est souvent présenté comme la meilleure façon de distinguer à l'intérieur d'un corps politique indifférencié ceux qui auront plus de droit que les autres.

Les textes les plus modernes intègrent la totalité des Espagnols dans le corps politique selon une procédure unique, valable pour tous. Sur l'ensemble des textes $40 \%$ proposent une organisation de la nation à partir d'individus égaux. Les textes qui cherchent à distinguer les élites à l'intérieur du corps politique peuvent être aussi considérés comme présentant la nation composée d'individus et non plus d'ordres. Ce sont alors $51 \%$ des textes qui font table rase de l'ancienne vision du corps politique.

La justification de cette rupture renvoie à la doctrine pactiste selon laquelle la communauté - la société - définit elle-même le régime et le chef qui la gouverneront, et ce dès l'origine. La crise du printemps 1808 a rompu, de fait, le cadre du pacte. À cette communauté tout entière revient le droit de se doter d'une nouvelle forme de régime pour recomposer le pouvoir en Espagne. C'est donc le peuple qui est appelé à participer à cette reconstitution, comme à l'origine des sociétés.

29 En 1808 c'est par l'intermédiaire d'une représentation populaire que peut se réaliser cette participation et par l'intermédiaire des élections que se matérialise l'inclusion de tous dans le corps politique.

Il existe d'autres visions plus modernes : plutôt que le pactisme, elles font référence aux Droits de l'homme comme la Révolution française a pu les définir. C'est Alvaro Florés Estrada, un des meilleurs représentants de la fin des Lumières espagnoles, qui les expose le plus clairement pour présenter sa constitution ${ }^{15}$. Comme les principes individuels des Droits de l'homme atomisent la société en renvoyant chacun de ses membres à ses propres intérêts - la sécurité, la liberté par exemple - il faut instituer un lien collectif qui permette de réunir tous ces intérêts particuliers en un intérêt général, possible puisque tous jouissent des mêmes droits. La participation de tous à 
l'élaboration de leur gouvernement constitue ce lien et fonde un corps politique actif producteur de la volonté générale ${ }^{16}$.

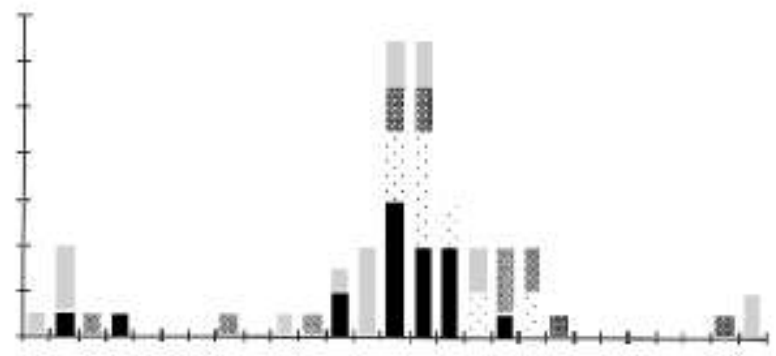

L'étude de la répartition dans le temps de ces différentes positions vis-à-vis de la constitution du corps politique, permet de mieux cerner l'influence des circonstances sur le débat. Le graphique ci-dessus présente la chronologie des textes selon leur définition du corps politique.

La vision moderne est très présente pendant l'été 1808. C'est le moment des premières réflexions sur le politique, nous sommes juste après le soulèvement et l'image du peuple actif marque les auteurs qui lui concèdent une participation entière dans la communauté politique et cherchent à fonder une représentation de la nation d'essence populaire. L'autre position représentée à cette période est la vision traditionnelle, correspondant à des auteurs qui veulent préserver certaines formes jugées valables pour atténuer le bouleversement subi par la monarchie espagnole.

Ensuite, lors de la consultation sur les Cortes nous voyons un éparpillement des opinions avec toute une gamme de propositions allant du plus traditionnel (en général les évêques), au plus moderne (en général les universités). Entre les deux, les juntes et les municipalités sont celles qui cherchent les solutions hybrides assurant au peuple une intégration franche dans le corps politique tout en évitant toutes dérives trop démocratiques. Tenants d'une autorité, mais en liaison avec l'élément populaire, ces institutions veulent reconstruire l'ordre politique à partir de l'expérience du soulèvement tout en réservant l'exercice du pouvoir aux cadres traditionnels de la société.

À l'approche de la réunion des Cortes nous pouvons nous rendre compte que les termes du débat ont changé. Les idées les plus avancées ont triomphé suite à la publication de la convocation du peuple aux Cortes extraordinaires. Les seuls textes recensés proviennent de particuliers qui prennent parti pour une participation prédominante du peuple parfois nuancée par le rôle supérieur assigné aux élites. Suivant en cela le règlement électoral (suffrage quasi universel) préparé par la Commission des Cortes, ils optent pour un corps politique constitué de citoyens.

Le domaine d'action du politique ; le problème de la souveraineté 

système du favori est attaqué particulièrement. Une des critiques qui revenaient le plus souvent dans les premiers textes de l'été 1808 était le «despotisme ministériel » et l'arbitraire des décisions du favori, en l'occurrence Godoy. De la même façon, l'altération de l'ordre dynastique était considérée comme la preuve de la décadence de la monarchie. Le recours aux lois fondamentales du royaume permet de placer les aménagements politiques dans le fil d'une reconstitution du régime dans la pureté de ses origines.

41 Poursuivant cette réflexion sur l'indépendance du corps politique par rapport à son prince, d'autres auteurs proposent que les Cortes soient totalement souveraines et que leur revienne la totalité du pouvoir de faire les lois. Faire les lois, tel que l'explique un texte de l'été 1808, c'est la formalisation de la volonté générale à travers les règles que se donne la communauté pour assurer son existence ${ }^{19}$. En tant que tel, le pouvoir législatif ne peut revenir qu'à la nation par l'intermédiaire des Cortes. Elles doivent se constituer par elle-même sans l'intervention du prince. Ensuite le prince gouverne, c'est-à-dire qu'il exécute les décisions prises par les Cortes. Nous nous trouvons face à une théorie différente basée sur l'unicité et l'indivisibilité de la souveraineté. Résidant dans la nation elle ne peut être partagée avec le prince ${ }^{20}$. Cette théorie, étrangère aux lois fondamentales, nécessite des explications et les auteurs passent souvent beaucoup de temps à définir les différents éléments, faisant un travail d'éducation politique, de pédagogie de la politique. la référence au pactisme est toujours présente dans le 
constat, point de départ de la réflexion : comme le pacte qui constituait la monarchie espagnole a été rompu, «la nation a récupéré la totalité de ces droits » et peut donc légitimement se reconstituer à travers de nouvelles Cortes.

L'objet des premières Cortes sera d'élaborer la constitution de la monarchie espagnole, et ceci sans intervention du roi. La constitution, qu'elle soit considérée comme le rassemblement des codes, des lois fondamentales adaptées aux nouvelles conditions, ou qu'elle soit un nouvel ensemble de règles correspondant au nouveau contrat de la communauté, est l'affirmation de cette autonomie conférée à la nation par l'intermédiaire du pouvoir législatif, défini comme étant à l'origine un pouvoir constituant ${ }^{21}$.

D'autres auteurs, à peu près la moitié des textes étudiés, ne s'embarrassent pas de justifications conciliant une certaine légalité historique avec les nouvelles circonstances. Refusant de voir dans les lois fondamentales autre chose qu'un rassemblement vague de lois aujourd'hui inutiles $\left({ }^{22}\right)$, refusant d'y voir un code qui tempérait le pouvoir du roi, ils plaident pour une reconstruction totale et radicale $\mathrm{du}$ régime.

«Les maux des sociétés, en fin de compte, n'ont d'autres causes que l'oubli ou le mépris des droits des citoyens et le manque de respect des devoirs du gouvernement vis-à-vis des gouvernés.» ${ }^{23}$

La nation n'a pas à se justifier, son pouvoir existe, elle l'avait perdu tant par sa faiblesse que par la malignité des princes.

Ces positions radicales affirment l'autonomie du corps politique, à partir des droits premiers de ses membres. La référence n'est plus le pactisme mais le principe du contrat social passé entre des individus isolés décidant de s'associer pour pouvoir jouir de leurs droits naturels. La nation est "essentiellement souveraine " et non plus seulement originellement souveraine (théorie pactiste). Il est remarquable de noter que ce genre de propositions émane surtout de particuliers écrivant plutôt vers 1810. Ils tirent les conclusions logiques d'une place de plus en plus importante accordée au peuple comme source de légitimité aussi bien à travers les dernières décisions de la Junte centrale (ce que l'on appelle la « victoire» du groupe « libéral » de Quintana $\left({ }^{24}\right)$ ) que par l'exemple de la formation de la deuxième junte de Cadix dont les membres sont élus par l'ensemble de la population masculine de la ville : la légitimité n'émane que de la nation

L'élection comme réalisation de la souverainetéL'impératif de la représentation de la nation

Les nouvelles définitions de la nation et les précisions données sur le type de pouvoir $\mathrm{du}$ corps politique constitué amènent à traiter des moyens de réaliser cette souveraineté. Le pouvoir de la nation ne peut s'exercer directement en raison de la nature de ce corps. Il s'incarne à travers sa représentation. Toute souveraineté nationale est d'essence représentative ${ }^{25}$. Le premier acte du corps politique, celui qui le fait exister, correspond au choix de ses représentants. Selon les bornes fixées à ce corps politique, le type de représentation sera différent.

La vision traditionnelle propose une représentation nationale très proche de l'image historique des anciennes Cortes: ce sont les ordres qui seront représentés. Certains partent des villes privilégiées qui avaient droit de vote aux Cortes et proposent qu'on élargisse ce privilège à d'autres villes importantes ${ }^{26}$. D'autres prennent pour base les diocèses ${ }^{27}$. Plus rarement les provinces ${ }^{28}$. Si le résultat aboutit pour chaque cas à une 
représentation éclatée entre les prélats, la noblesse et le peuple, les moyens ne sont jamais identiques. Pas un de ces projets ne propose la même méthode mais il en ressort toujours que le choix lors de l'élection se trouve limité par la permanence des ordres. Parfois même à l'intérieur de chaque ordre. La mention fréquente des prélats comme représentants de l'ensemble du clergé renvoie au système ancien où l'élite représente l'ensemble du corps. De la même façon, les Grands d'Espagne sont mieux placés pour représenter la noblesse. Pour ce qui est de la représentation du tiers, les auteurs raisonnent à partir des villes et de leur municipalité : le peuple est représenté par l'intermédiaire de la municipalité. Représentation de type ancien puisque le choix est limité à l'élite municipale constituée en corps et que l'ensemble des habitants (vecinos) ne paraît pas convoqué dans ces cas.

Tout en conservant cette vision deux textes proposent de pallier le déficit de représentativité des villes aux Cortes. Pour les villes ayant déjà ce droit, on ne modifie pas le mode de choix de la représentation, mais pour les autres on institue un nouveau système de représentation à partir de l'ensemble des habitants de la ville. On calcule le nombre de députés en rapport avec la population de ces villes ${ }^{29}$. Ce mélange de respect de la tradition et d'idées nouvelles correspond à une certaine vision du patriotisme où la tradition est considérée comme la référence première mais où quelques innovations sont tentées en parallèle pour être en accord avec les circonstances.

D'autres hybridations entre tradition et nouveautés sont repérables parmi les projets. Prenons l'exemple du rapport de la municipalité de Grenade qui après avoir fustigé les anciennes lois et les Cortes traditionnelles, ne croit pas opportun pour autant que la représentation nationale soit basée sur des individus égaux. On garde la distinction d'ordre mais pour être en accord avec la réalité du pays et pour qu'aucune catégorie ne soit lésée, on élit les représentants de chaque ordre en proportion du nombre de leurs membres. Ce maintien de la séparation en ordres est motivé par la crainte de la démocratie, mais nous pouvons nous rendre compte de l'aspect strictement formel de cette protection anti-démocratique, puisque finalement les représentants sont élus proportionnellement à l'ensemble de la population et que les ordres sont considérés égaux entre eux.

D'autres exemples de transition vers la représentation moderne, basée sur le libre choix d'un individu par l'ensemble des membres du corps politique, privilégient tel ou tel aspect de la tradition et de la nouveauté. L'élément nouveau qui se rencontre le plus souvent est la proportionnalité des représentants par rapport à la population. La junte de Palma de Majorque adopte ce principe mais en même temps fait élire deux députés pour chaque province, permanence d'une représentation de corps (la province) et en même temps confère une place de député de droit à chaque archevêque d'Espagne et d'Amérique. La junte de Valence penche aussi vers un système double où l'on représente la nation de deux façons différentes, l'une en la considérant comme constituée par l'ensemble des provinces (deux députés pour chaque capitale de province, c'est-à-dire que la capitale représente l'ensemble de la province), et comme constituée par l'ensemble des habitants (un député élu pour 300000 habitants). Il n'est pas étonnant de retrouver ce système double élaboré par les juntes provinciales. La pratique de leur pouvoir depuis le soulèvement les a placées à la tête de leur province. C'est en tant que représentantes de la province qu'elles ont envoyé deux de leurs membres comme députés à la Junte centrale. 
51 Le principe d'une représentation proportionnelle à la population correspond à tous les projets les plus modernes. Les termes individus et citoyens sont cités pour définir ceux qui prendront part à l'élection. Ce sont 31 textes, c'est-à-dire pratiquement la moitié, qui proposent ce type de représentation.

L'arithmétique politique : les calculs sur la représentation

52 Les projets de représentation moderne comportent tous la description du système électoral qui correspondrait le mieux à la composition du corps législatif.

Une représentation proportionnelle au nombre d'habitants nécessite tout d'abord de fixer le taux de représentativité. La question du nombre de députés détermine ce taux. Un corps législatif nombreux n'a pas le même sens politique que s'il est restreint. Les taux se situent entre 1 pour 30000 et 1 pour 60 000. Les auteurs prennent comme base de calcul le dernier recensement : 11 millions d'habitants pour la péninsule et à peu près autant pour les possessions d'outre-mer. La taille du corps législatif varie entre les différents projets selon le taux adopté et selon qu'on intègre ou non l'Amérique dans la représentation ( et la plupart des textes n'en parlent pas sauf lors de la consulta al pais où un des points traitait de sa participation aux Cortes - les réponses acceptent rarement une représentation égale, le plus souvent une représentation symbolique). Les chiffres extrêmes sont 733 députés pour le projet de cortes les plus nombreuses et 183 pour les plus restreintes.

54 Les partisans d'une représentation nombreuse la justifient en pensant que les décisions de l'assemblée correspondraient plus sûrement à l'expression de la volonté générale et que le grand nombre de députés rendrait difficile au pouvoir exécutif de la soumettre. Mais en même temps certains estiment que des Cortes trop nombreuses seraient trop difficiles à gérer et qu'inévitablement des « factions » apparaîtraient ${ }^{30}$. L'exemple de la France est cité, les "dérapages » de la Révolution sont expliqués par l'agitation qui régnait dans les assemblées trop nombreuses. Il s'avère que les partisans de Cortes peu nombreuses (moins de 400 députés) veulent éviter que le nouveau système soit trop démocratique en donnant une représentativité du peuple trop importante. À l'inverse, des Cortes comportant plus de 400 députés affirment la prééminence de la représentation populaire.

55 Après avoir réglé la question du nombre des députés, les projets s'intéressent au mode d'élection. Nous trouvons là des nuances quant au sens à donner à la représentativité de la nation.

Un seul texte propose un scrutin organisé à partir des livres des contributions évitant de convoquer la population à une assemblée électorale. Les électeurs se succéderaient pour apposer leur choix en face de leur nom. Ce système est pensé pour éviter le « tumulte » de l'assemblée et non pas pour garantir l'individualité du choix. En effet le type de représentation proposée par l'auteur est celle de Cortes divisées selon les trois ordres avec un cens très élevé pour être député du tiers (15000 réaux $)^{31}$.

Les autres présentations de mode d'élection choisissent un suffrage à deux voire trois degrés. Le schéma du système électoral est le suivant : la base électorale est la paroisse, les habitants y sont assemblés pour choisir leur électeur qui ensuite ira se joindre aux autres électeurs à la capitale de la province (dans le cas du suffrage à trois degrés, il y a une étape intermédiaire entre la paroisse et la capitale: le siège du corregimiento [district]). À partir de cette trame, il existe de nombreuses modalités de vote. Plusieurs auteurs s'appuient sur le système des élections des diputados del común des 
municipalités pour choisir les « commissaires électeurs » qui concourront à la capitale de province. Un autre terme pour désigner les électeurs issus du premier degré revient souvent: celui de procureurs. Nous voyons là une solution de continuité avec les quelques pratiques électorales qui existaient en Espagne depuis la réforme d'Aranda sur les municipalités en 1766 (élection annuelle de représentants des habitants dans le cadre de la paroisse).

Quelle que soit la manière de procéder, il est manifeste que le premier degré de l'élection opère un tri dans l'électorat. Au second degré nous ne devons retrouver que les personnes les plus capables de choisir ceux qui iront assumer le pouvoir législatif. Il n'est jamais question de demander à l'ensemble du peuple de se positionner par rapport à un choix de politique, de choisir un parti plutôt qu'un autre, mais seulement de déléguer leur portion de souveraineté à d'autres qui la représenteront au nom de toute la nation. Donc pas de campagne électorale, les consignes de vote se limitent à la préférence qui doit être donnée aux meilleurs paroissiens :

«Une réputation de probité et d'instruction est le seul guide que l'on suivra lors de ces élections ou nominations.» ${ }^{32}$

Il est manifeste aussi que ces élections au premier degré doivent renvoyer l'image d'une nation unie, et que ses représentants doivent montrer l'unanimité qui seule assure la cohésion de tout corps. Dans les circonstances de la lutte patriotique, ce besoin d'unanimité correspond aussi bien à une permanence de la vision des élections anciennes qu'à une nécessité stratégique.

Certains auteurs proposent à l'issue du scrutin un tirage au sort pour désigner le ou les députés. Pratique traditionnelle qui fait intervenir la Providence dans le choix des individus, mais aussi rempart contre toute manipulation de l'électorat. Il s'agit d'éviter les factions, hantise des patriotes qui basent leur engagement sur un idéal d'unanimité.

61 Par ailleurs, et dans le même esprit qui tend à raccorder pratiques anciennes et modernes, nous trouvons fréquemment la mention de festivités pour marquer l'élection. Fêtes traditionnelles avec illumination et processions accompagnées de rogations pour que le peuple mesure la gravité de l'acte et pour que Dieu les guide dans leur choix. Plus civiquement, d'autres textes réclament simplement un discours du curé de la paroisse et du syndic-procureur du commun puis un serment public des électeurs choisis pour représenter les paroissiens.

Les projets s'intéressent ensuite aux conditions d'éligibilité. Il s'agit, là aussi, d'un moyen de préciser la réalité de cette représentation populaire. La plupart des textes consacre du temps à la présentation des conditions d'éligibilité, surtout quand elles sont restrictives ou favorisent une catégorie plutôt qu'une autre. Pour le premier degré, malgré les principes généreux sur la représentation large du peuple $\left.{ }^{33}\right)$, certains auteurs limitent le droit de vote par la richesse ou la possession d'une propriété. Les cens sont généralement bas, permettant tout de même au plus grand nombre de participer à l'élection. En revanche, en ce qui concerne les électeurs du deuxième degré, les restrictions sont plus importantes. Le cens est relevé. L'argent est loin d'être le seul critère pour définir le profil de l'électeur du deuxième degré. Des auteurs critiquent la restriction par l'argent en craignant qu'elle n'exclue de l'élection des personnes de «talents » mais pas assez riches. Nous retrouvons la question du « marc d'argent» qui avait divisé les constituants français en 1791. Ils souhaitent voir l'assemblée composée des individus les plus éclairés de l'Espagne, dans l'optique d'une réforme politique de la monarchie, mais aussi selon le principe de la délégation du 
pouvoir aux plus capables de l'exercer. Nous retrouvons aussi, sous-entendu, une certaine défiance de nos auteurs vis-à-vis du peuple : il est encore "ignorant ", n'a pas atteint le niveau de connaissance politique lui permettant de participer aux affaires publiques.

La question des talents oblige certains auteurs à des acrobaties dans les calculs de la représentation. Ainsi, on considère que l'on trouve plus de ces personnes dans les grandes villes où abondent les établissements publics (collèges, universités, tribunaux) mais aussi les lieux de réunion de l'élite éclairée (tertulias, sociétés économiques). On cherche donc à les avantager en leur donnant un taux de représentativité supérieur au reste de la province. F. X. Uriortua dans sa Tentativa para variar la representación nacional propose que les villes de Madrid, Barcelone et Séville aient au moins deux députés de plus en raison de leur importance ainsi que les villes de Cadix, Málaga et même Xérez de la Frontera en raison des relations commerciales internationales qu'elles entretiennent et donc de leur utilité pour le pays. De la même façon, on conseille de faire en sorte que les nobles et les ecclésiastiques soient préférés aux autres électeurs mais sans institutionnaliser un privilège électoral. Là aussi, le rôle des villes capitales de province est rehaussé puisque c'est dans ces villes que réside le plus de membres de la noblesse. Plusieurs systèmes sont ainsi élaborés pour ne garder dans les Cortes que la sanior pars de la nation. Ces limites à l'égalité du corps politique montrent la différence qui est faite entre la souveraineté déclarée du peuple et son exercice. Le peuple n'est convoqué que pour faire un choix, le meilleur possible. Le pouvoir réel réside dans l'élite ainsi dégagée. Selon le type de réformes que l'on assigne à la future assemblée, les auteurs privilégient l'élite économique ou bien l'élite intellectuelle. L'élite économique, dans un esprit proche des physiocrates, saura choisir les réformes qui favoriseront le commerce et l'industrie et moderniseront l'agriculture. L'élite intellectuelle permettra la mise en place d'un système politique qui garantira l'Espagne d'un retour au despotisme. Il apparaît clairement que dans ce cas la vision idéale des membres des Cortes renvoie aux modèles de réformes élaborés depuis le règne de Charles III dans les cercles éclairés comme ceux des Sociétés des amis du pays. Ce moment où tout est à construire est l'occasion pour tenter de les appliquer. Ces esprits formés par la critique de l'absolutisme excluent de l'éligibilité les fonctionnaires de l'administration royale et tous les bénéficiaires de pensions royales. Leur dépendance vis-à-vis du pouvoir exécutif est considérée comme incompatible avec le rôle de contrôle du pouvoir royal. Pourtant un auteur défend les fonctionnaires royaux au nom de leurs connaissances des affaires et de leur niveau d'instruction supérieur à la moyenne ${ }^{34}$. L'utilité publique nécessite leur présence dans l'assemblée car le nouveau système n'en fait plus des hommes au service du roi mais au service de la nation toute entière.

Les autres exclusions du vote ou de l'élection touchent ceux qui sont trop liés à un maitre ou à un groupe : cas des domestiques et du clergé régulier (un texte propose même que chaque communauté religieuse ait droit à une seule voix collective). Enfin, et selon la même logique qui écarte du droit de vote les personnes dépendantes, les femmes, comme les mineurs, ne sont pas autorisées à participer aux élections.

Conclusion la particularité de la révolution espagnole

La multiplicité des programmes, des calculs démontre l'intérêt des auteurs pour la question des pratiques électorales. Ils cherchent à composer un système parfait en utilisant plusieurs principes, en revisitant certaines pratiques anciennes et en adoptant 
d'autres modernes. Les références foisonnent et s'entremêlent. L'utilisation de l'histoire de l'Espagne, en particulier le Moyen Âge est un élément souvent rhétorique, alors que les réflexions sur la souveraineté rappellent les débats de la Révolution française. Dans une certaine mesure, la problématique du passage d'un souverain-roi à un souverain-peuple rapproche les années françaises 1789-1792 des années 1808-1810. La richesse de la pensée politique patriote correspond à cette conjonction entre les circonstances qui obligent la recherche de nouvelles formes politiques, le ressentiment vis-à-vis d'un système absolutiste qui a fait faillite et l'apparition du peuple sur la scène politique comme acteur (ou comme décor). Les différences entre chaque projet sont le fruit de cette période de transition entre l'Ancien Régime et la modernité politique. Nous avons pu parcourir toute une échelle de propositions allant d'un aménagement de la tradition à une volonté de rupture. Les degrés ne sont pourtant pas linéaires et c'est un des enseignements de l'analyse de détails des pratiques électorales projetées. Il en ressort une impression d'hybridation complexe qui fait la fécondité des périodes de transition ${ }^{35}$. Chaque auteur apporte sa contribution personnelle à l'édifice de reconstruction d'une monarchie en crise. L'élargissement du débat à la sphère publique est une autre donnée essentielle de la période l'apparition de l'opinion comme action politique rationnelle. Ces éléments, joints au refus de solutions dictées de l'extérieur, fondent une des particularités de la révolution espagnole.

\section{NOTES}

1.Cf. Richard HOCQUELLET, « Los reinos en orfandad, la formación de las juntas supremas en España (1808), Los procesos de las independencias en la América española, Actas del Congreso internacional, Colegio de Michoacán-Instituto Nacional de Antropología e Historia, Morelia (Mexico), 2000.

2.Cf. Patrice GUENIFFEY, Le nombre et la raison. La Révolution française et les élections, Paris, Éditions de l'E.H.E.S.S., 1993, p.27.

3.Tous les rapports (dictamen) disponibles sont conservés à l'Archivo del Congreso de los Diputados (A.C.D.) de Madrid, série General, legajos 5 et 6.

4.L'ensemble de la consulta fait l'objet d'une analyse détaillée dans ma thèse $D u$ soulèvement patriotique à la souveraineté nationale. La première phase de la révolution espagnole, 1808-1810 (Université de Paris I, 1999), pp.559-582. Une version abrégée est à paraître en 2001 à la Boutique de l'Histoire Éditions Résistance et révolution durant l'occupation napoléonienne, Espagne 1808-1812.

5.Il s'agit de 6 textes tirés des correspondances de la junte de Catalogne, Archivo de la Corona de Aragon (A.C.A.), Junta de Cataluña, caja 11.

6.Ces écrits proviennent des deux immenses collections de textes patriotiques conservées à Madrid: la Colección documental del Fraile déposée au Servicio Histórico Militar et la collection de Gómez-Imaz déposée à la Biblioteca Nacional.

7.Manifestación política sobre las actuales circunstancias, Valence, 10 juin 1808.

8.Proclama de Belalcazar, dans Manifiestos firmados por distintas personalidades, Séville 1808. 
9.Richard HOCQUELLET, « La recomposition politique et territoriale de l'Espagne pendant la Guerre d'Indépendance, Voies de renouvellement de l'histoire de l'Empire, colloque Université d'Avignon, mai 2000. Actes à paraître à la Boutique de l'Histoire Éditions. 10.Cf. les analyses de Jean-Yves GUIOMAR, La Nation entre l'histoire et la raison, Paris, La Découverte, 1990, pp.116 et 120 .

11.À ce titre, il serait réducteur de considérer le mouvement patriotique comme « un nationalisme du ressentiment. L'histoire des Espagnols n'est pas totalement déterminée par les actes de Napoléon. Natalie PETITEAU, Napoléon, de la mythologie à l'histoire, Paris, Seuil, 1999, pp.292-293.

12.Dictamen de la Junta de Badajoz, dans Miguel ARTOLA, Los origenes de la España contemporánea, Madrid, 1959, t. II, p.294.

13.Dictamen de l'Audience d'Estrémadure, 25-X-1809, dans Fedérico SUAREZ (ed.), Informes oficiales sobre Cortes, Pampelune, Universidad de Navarra.

14. Voir le texte de F. X. URIORTUA, Tentativa sobre la necesidad de variar la representación nacional que se ha de convocar a las futuras Cortes, op. cit. et la réponse de F. de Paadín, (BN Madrid R 60087).

15.Alvaro FLORES ESTRADA, Constitución para la Nación española, Birmingham, 1810.

16. Voir les analyses de Pierre RosanVALLON, dans Le sacre du citoyen, histoire du suffrage universel en France, Paris, Gallimard, 1992, pp.55-63 « L'égalité politique et les formes du social».

17.Voir la théorie élaborée par la néo-scolastique espagnole du droit à la rébellion en cas de manquement du prince au pacte.

18.Dictamen de la Junte de Majorque in Informes oficiales sobre Cortes, op. cit., vol.1, p.114. 19.« La loi n'est rien d'autre que l'expression de la volonté et du consentement de la nation, manifestée par elle-même ou par le moyen de ses représentants, et publiée de manière à ce que personne ne puisse l'ignorer. Pensamientos de un patriota español para evitar los males de una anarquía o la división entre las provincias que actualmente componen el territorio de toda la monarquía española y establecer un gobierno conveniente a toda ella, observando lo que prescriben la justicia, la razón natural y los derechos de la Nación misma, anonyme, juillet 1808, p.10.

20.Le débat sur la souveraineté partagée entre le prince et les Cortès ou sur l'unicité de la souveraineté des Cortès sera largement développé lors des Cortes de Cadix. Voir Joaquín VARELA SUANZES, La teoría del Estado en los origenes del constitucionalismo hispánico (las Cortes de Cádiz), Madrid, Centro de Estudios constitucionales, 1983, chapitre 2.

21.La revendication de Cortes constituantes s'impose progressivement. Cf. Francisco TOMAS Y VALIENTE, « Genesis de la Constitución de 1812. De muchas leyes a una sola constitución, Anuario de de Historia del derecho español, Madrid, 1996, pp.13-125.

22.Le dictamen de l'université de Séville parle de « lois vagues et traditionnelles s'affaiblissant petit à petit et qui finalement furent oubliées pendant le dépostime des rois. À Grenade, la municipalité se référant aux vieux codes de lois considère, " qu'aujourd'hui, ils sont tous trop particuliers ou inutiles et qu'ils ne doivent pas distraire des sérieuses et graves occupations du royaume. Les mots les plus durs reviennent au texte: Reflexiones sociales ó ideas para la constitución española que un patriota ofrece a los representantes de Cortes (Valence, 1810) qui parle des Partidas comme d'un " mélange extravagant de liberté et de servitude, de savoir et d'ignorance, et assure qu'elles ne forment pas la constitution de l'Espagne (p.3).

23.Alvaro FLORES ESTRADA, introduction à la Constitutión para la nación española, op. cit. 
24.Dans la précipitation du départ de la Junte centrale de Séville à Cadix, début février 1810 , seul le décret de convocation des députés du tiers a été envoyé. Certains accusent Quintana, premier officier du secrétariat, d'avoir volontairement fait disparaittre les décrets de convocation de la noblesse et du clergé sans qu'il soit possible de le vérifier d'après les sources (voir le récit dans Albert DEROZIER, Quintana ou la naissance $d u$ libéralisme espagnol, Besançon, Les Belles Lettres, 1968, pp.497-498 et Federico SUAREZ, El proceso de la convocatoria a Cortes 1808-1810, Pampelune, Eunsa, 1982, pp.427-438).

25. Marcel GAUCHET, La révolution des pouvoirs. La souveraineté, le peuple et la représentation, 1789-1799, Gallimard, Paris, 1995, p.47.

26.Voir les dictamenes de l'évêque d'Albarracín, de la ville de Cordoue (A.C.D., leg.6), mais aussi les Cartas Patrióticas du père Tomás de Salas (Séville, 1809) et la Memoria de H. M. Cipriano envoyée à la Junte centrale fin 1808 à propos des Cortes (Madrid, Archivo Histórico Nacional, leg.52-D, $\mathrm{n}^{\circ} 183$ ).

27.Dictamenes des évêques de Ciudad Rodriguo ou de Cordoue (A.C.D., leg.6).

28.Dictamen de l'Audience d'Estrémadure (A.C.D., leg.5), Manifiesto histórico sobre las actuales circunstancias publié à Valence en 1808 ou mémoire de l'archidiacre de Vich de septembre 1809 (A.C.A.).

29.Le dictamen de Cordoue propose qu'on élise un député pour chaque ville de plus de 4000 habitants et que pour les villes de moins de 4000 un député sans droit de vote aux Cortes soit nommé (observateur appelé defensor). Un article du Voto de la Nacion Española du 3 janvier 1810 demande que l'on garde les trois classes habituelles pour la convocation des Cortes, les villes ayant voix aux Cortes gardent un député auquel on en adjoint un second pour chaque junte provinciale, et pour le reste on élit un député pour 40000 à 50000 familles.

30.« De los gobiernos representativos, dans El Espectador Sevillano, n67, op. cit.

31.Mémoire envoyé à la junte de Catalogne par Juan Gispert, 30 août 1809, (A.C.A.).

32.Tentativa sobre la necesidad de variar la representación nacional... op. cit., p.23.

33.Le suffrage le plus élargi ne peut être à cette époque que celui des hommes majeurs (l'âge de la majorité varie selon les auteurs entre 21 et 27 ans).

34.Carta que D. F. de P. de Paadín escribía al señor D. F. X. Uriortua exáminando su papel intitulado Tentativa sobre la necesidad de variar la representación nacional que se ha de convocar en las futuras Cortes, Cadix, 1810.

35.Cf. François-Xavier GuERRA, Modernidad e independencias, Mapfre, Madrid, 1992, pp. 170-175.

\section{RÉSUMÉS}

Alors que l'Espagne est déchirée par la guerre contre les troupes napoléoniennes, les patriotes qui refusent les abdications des Bourbons en faveur de Joseph Bonaparte tentent d'organiser un pouvoir souverain légitime. Le versant politique de la résistance comporte un moment crucial le débat sur la convocation d'une assemblée assurant la représentation de la nation en lutte. Spontanément ou à la demande du gouvernement central patriote, des particuliers et des institutions vont élaborer des projets électoraux. L'ensemble de ces contributions forme une 
série d'énoncés politiques d'une grande richesse pour saisir la complexité d'une période de transition entre l'Ancien Régime et la modernité politique car leurs auteurs s'obligent à transposer dans la pratique des théories. Inversement l'analyse de ces projets renseigne sur la réalité et la validité des idées ainsi que leurs implications concrètes. Trois thèmes sont ici privilégiés la définition du corps politique national, la question de la souveraineté et le problème de sa représentation. Même si l'hybridation des théories est la règle générale, le processus enclenché mène à la valorisation de la nation comme source unique de la légitimité et comme base de la souveraineté.

From Sujects to citizens. An analysis of electoral projects before the Cortes of Cadix, 1808-1810.

As Spain is engaged in the war against the napoleonic armies, the patriots who refuse the abdications of the Borbons on Joseph Bonaparte intent to organise a legitimate sovereign power. The political side of the struggle comprises a crucial moment : the debate for the convocation of a national assembly. From themselves or requested by the central patriot government, private persons or institutions are going to work on electoral projects. All of these texts compose a whole of great ressources political wordings. They help to understand the complexity of a period of transition between Ancien Régime and political modernity because the autors must transpose in pratices theories. Inversely, the analysis of these projects give informations on the ideaes' reality and validity as their concrete implications. We base this study on three themes : the definition of the political corps; the question of sovereignty and the problem of his representation. Even if theories' hybridizations are general, the process lead to the valorisation of the nation as unic source of legitimity and base of sovereingty.

INDEX

Mots-clés : Espagne, révolution, élections, nation

\section{AUTEUR}

\section{RICHARD HOCQUELLET}

Université de Paris I Panthéon-Sorbonne - UMR 85-65 